Submitted to the Annals of Applied Statistics

arXiv: math.PR/0000000

\title{
A STATISTICAL FRAMEWORK FOR DATA INTEGRATION THROUGH GRAPHICAL MODELS WITH APPLICATION TO CANCER GENOMICS
}

\author{
By Yuping Zhang, Zhengqing Ouyang and Hongyu ZhaO
}

University of Connecticut, The Jackson Laboratory for Genomic Medicine, and Yale University

\section{Supplementary 1. Proof of convexity.}

Proposition 1.1. The negative log pseudo-likelihood in our model is jointly convex in all the parameters $\left\{\lambda_{j}, \lambda_{j m}, \eta_{0}, \eta_{j}, \mathbf{\Phi}_{0}, \mathbf{\Phi}_{j}\right\}$ over the region $\Omega_{x r r}>0$.

Proof. To prove the convexity of $\ell(\Theta \mid \mathbf{x}, \mathbf{y})$, it is sufficient to verify that each term is convex.

1. $-\log P\left(x_{j} \mid \mathbf{x}_{\backslash j}, \mathbf{y} ; \boldsymbol{\Theta}\right)$ is jointly convex in $\lambda_{j}, \lambda_{j m}, \eta_{j}$ and $\boldsymbol{\Phi}_{j}$ because it is a multiclass logistic regression.

2. $-\log p\left(y_{r} \mid \mathbf{y} \backslash r, \mathbf{x} ; \boldsymbol{\Theta}\right)$ is jointly convex in $\eta_{0}, \eta_{j}, \mathbf{\Phi}_{0}$ and $\boldsymbol{\Phi}_{j}$. This is because the following facts hold.

(a) $-\frac{1}{2} \log \Omega_{x r r}$ is a convex function.

(b) $\frac{\Omega_{x r r}}{2}\left(\frac{\eta_{0 r}+\sum_{j=1}^{p} \eta_{j r}\left(x_{j}\right)-\sum_{s \neq r} \Omega_{x r s} y_{s}}{\Omega_{x r r}}-y_{r}\right)^{2}$ is convex because the fact that $g(u, v)=\frac{v}{2}\left(\frac{u}{v}-c\right)^{2}$ is convex. Let $v=\Omega_{x r r}, u=\eta_{0 r}+$ $\sum_{j=1}^{p} \eta_{j r}\left(x_{j}\right)-\sum_{s \neq r} \Omega_{x r s} y_{s}$, and $c=y_{r}$. Notice that $y_{s}, y_{r}$ and $x_{j}$ are fixed and $u$ is affinely related to $\eta_{0 r}, \eta_{j r}$ and $\Omega_{x r s}$. Thus, the convexity for $\frac{\Omega_{x r r}}{2}\left(\frac{\eta_{0 r}+\sum_{j=1}^{p} \eta_{j r}\left(x_{j}\right)-\sum_{s \neq r} \Omega_{x r s} y_{s}}{\Omega_{x r r}}-y_{r}\right)^{2}$ holds because that a convex function composed with an affine map is still convex.

To jointly analyze the graphical model for multiple classes, we need to minimize the additive negative log pseudo-likelihood, i.e. 


$$
\begin{aligned}
& \mathcal{L}\left(\boldsymbol{\Theta}^{(1)}, \cdots, \boldsymbol{\Theta}^{(K)} \mid \mathbf{x}^{(1)}, \cdots, \mathbf{x}^{(K)}, \mathbf{y}^{(1)}, \cdots, \mathbf{y}^{(K)}\right) \\
& =\sum_{k=1}^{K} \ell\left(\boldsymbol{\Theta}^{(k)} \mid \mathbf{x}^{(k)}, \mathbf{y}^{(k)}\right) \\
& =-\sum_{k=1}^{K}\left(\sum_{j=1}^{p} \log P\left(x_{j}^{(k)} \mid \mathbf{x}_{\backslash j}^{(k)}, \mathbf{y}^{(k)} ; \boldsymbol{\Theta}^{(k)}\right)+\sum_{r=1}^{q} \log p\left(y_{r}^{(k)} \mid \mathbf{x}^{(k)}, \mathbf{y}_{\backslash r}^{(k)} ; \boldsymbol{\Theta}^{(k)}\right)\right)
\end{aligned}
$$

Proposition 1.2. The additive negative log pseudo-likelihood $\mathcal{L}\left(\left\{\boldsymbol{\Theta}^{(k)}\right\} \mid\left\{\mathbf{x}^{(k)}\right\},\left\{\mathbf{y}^{(k)}\right\}\right)$ is jointly convex in all the parameters $\left\{\boldsymbol{\Theta}^{(k)}\right\}$ over the region $\left\{\Omega_{x r r}^{(k)}>0\right\}$.

Proof. It immediately holds since the sum of convex functions is still convex.

Supplementary 2. Algorithm. We adapt the alternating direction method of multipliers (ADMM) in (Boyd et al., 2011) to solve our problem. Specifically, we have the following steps:

(a) Initialize the variables $\boldsymbol{\Theta}^{(k)}=0, \mathbf{U}^{(k)}=0$ and $\mathbf{Z}^{(k)}=0$ for $k=$ $1, \ldots, K$.

(b) Choose a scalar $d>0$.

(c) For $i=1,2,3, \ldots$, do the following until convergence:

(i) For $k=1, \ldots, K$, update $\boldsymbol{\Theta}_{(i)}^{(k)}$ as

$$
\boldsymbol{\Theta}_{(i)}^{(k)} \leftarrow \arg \min _{\boldsymbol{\Theta}^{(k)}}\left\{\ell\left(\boldsymbol{\Theta}^{(k)}\right)+\frac{d}{2}\left\|\boldsymbol{\Theta}^{(k)}-\mathbf{Z}_{(i-1)}^{(k)}+\mathbf{U}_{(i-1)}^{(k)}\right\|_{F}^{2}\right\} .
$$

We use a coordinate-wise descent algorithm to obtain each parameter in $\boldsymbol{\Theta}_{(i)}^{(k)}$.

Let $\mathbf{J}^{(k)}$ denote the matrix that contains the first order partial derivatives of $\ell\left(\boldsymbol{\Theta}^{(k)}\right)$ with every parameter in $\boldsymbol{\Theta}^{(k)}$ respectively. Let $\theta^{(k)}$ indicate any individual parameter in $\boldsymbol{\Theta}^{(k)}$, and let $z_{(i-1)}^{(k)}$ and $u_{(i-1)}^{(k)}$ indicate the corresponding elements in $\mathbf{Z}_{(i-1)}^{(k)}$ and $\mathbf{U}_{(i-1)}^{(k)}$ respectively. We use the proximal gradient method to obtain $\theta_{(i)}^{(k)}$, i.e. $\theta_{(i)}^{(k)} \leftarrow \operatorname{prox}_{t g}\left(\theta_{(i-1)}^{(k)}-t \mathbf{J}^{(k)}\left(\theta_{(i-1)}\right)\right)$, where $g(\theta)=\left\|\theta-z_{(i-1)}^{(k)}+u_{(i-1)}^{(k)}\right\|_{2}^{2}$, and $\mathbf{J}^{(k)}(\theta)$ indicates the first order 
partial derivatives of $\ell\left(\boldsymbol{\Theta}^{(k)}\right)$ at $\theta$. The proximal gradient algorithm is as follows.

Given $\theta_{(i-1)}, t_{(i-1)}$ and parameter $\beta \in(0,1)$

Let $t \leftarrow t_{(i-1)}$.

\section{Repeat until convergence}

$$
\begin{aligned}
& \text { 1. Let } v \leftarrow \operatorname{prox}_{t g}\left(\theta_{(i-1)}^{(k)}-t \mathbf{J}^{(k)}\left(\theta_{(i-1)}\right)\right) \\
& =\frac{1}{d+t}\left(d\left(z_{(i-1)}^{(k)}-u_{(i-1)}^{(k)}\right)+t\left(\theta_{(i-1)}-t \mathbf{J}^{(k)}\left(\theta_{(i-1)}\right)\right)\right)
\end{aligned}
$$

2. Update $t \leftarrow \beta t$.

Return $t_{(i)} \leftarrow t, \theta_{(i)} \leftarrow v$.

A typical value for the line search parameter $\beta$ is $1 / 2$.

(ii) For $k=1, \ldots, K$, update $\mathbf{Z}_{(i)}^{(k)}$ as

$$
\mathbf{Z}_{(i)}^{(k)} \leftarrow \arg \min _{\mathbf{Z}^{(k)}}\left\{\frac{d}{2} \sum_{k=1}^{K}\left\|\mathbf{Z}^{(k)}-\left(\Theta_{(i)}^{(k)}+\mathbf{U}_{(i-1)}^{(k)}\right)\right\|_{F}^{2}+P(\mathbf{Z})\right\} .
$$

(iii) For $k=1, \ldots, K$, update $\mathbf{U}_{(i)}^{(k)} \leftarrow \mathbf{U}_{(i-1)}^{(k)}+\left(\boldsymbol{\Theta}_{(i)}^{(k)}-\mathbf{Z}_{(i)}^{(k)}\right)$.

To solve (2.2), let $\mathbf{A}^{(k)}=\mathbf{\Theta}_{(i)}^{(k)}+\mathbf{U}_{(i-1)}^{(k)}$, then (2.2) can be rewritten as

$$
\mathbf{Z}_{(i)}^{(k)} \leftarrow \arg \min _{\mathbf{Z}^{(k)}}\left\{\frac{d}{2} \sum_{k=1}^{K}\left\|\mathbf{Z}^{(k)}-\mathbf{A}^{(k)}\right\|_{F}^{2}+P(\mathbf{Z})\right\} .
$$

The algorithm for problem (2.3) depends on the choice of penalty $P$. If $P$ is the fused lasso penalty, we have

$$
\left\{\mathbf{Z}_{(i)}^{(k)}\right\} \leftarrow \arg \min _{\left\{\mathbf{Z}^{(k)}\right\}}\left\{\frac{d}{2} \sum_{k=1}^{K}\left\|\mathbf{Z}^{(k)}-\mathbf{A}^{(k)}\right\|_{F}^{2}+\rho_{1} \sum_{k=1}^{K} \sum_{u \in C} \sum_{v \in C}\left|Z_{u v}^{(k)}\right|+\rho_{2} \sum_{k<k^{\prime}} \sum_{u \in C} \sum_{v \in C}\left|Z_{u v}^{(k)}-Z_{u v}^{\left(k^{\prime}\right)}\right|\right\} .
$$

We note that (2.4) is separable with respect to each pair of elements $(u, v)$ in matrix $\mathbf{Z}^{(k)}$. Thus, it can be further decomposed. For each $(u, v)$ we have the following optimization problem:

$$
\min _{\left\{Z_{u v}^{(k)}\right\}}\left\{\frac{d}{2} \sum_{k=1}^{K}\left(Z_{u v}^{(k)}-A_{u v}^{(k)}\right)^{2}+\rho_{1} \sum_{k=1}^{K}\left|Z_{u v}^{(k)}\right|+\rho_{2} \sum_{k<k^{\prime}}\left|Z_{u v}^{(k)}-Z_{u v}^{\left(k^{\prime}\right)}\right|\right\}
$$


It can be solved using the algorithm of the fused lasso signal approximator in (Hoefling, 2010).

If $P$ is the grouped lasso penalty, then problem (2.2) takes the form

$\left\{\mathbf{Z}_{(i)}^{(k)}\right\} \leftarrow \arg \min _{\left\{\mathbf{Z}^{(k)}\right\}}\left\{\frac{d}{2} \sum_{k=1}^{K}\left\|\mathbf{Z}^{(k)}-\mathbf{A}^{(k)}\right\|_{F}^{2}+\rho_{1} \sum_{k=1}^{K} \sum_{u \in C} \sum_{v \in C}\left|Z_{u v}^{(k)}\right|+\rho_{2} \sum_{u \in C} \sum_{v \in C} \sqrt{\sum_{k=1}^{K} Z_{u v}^{(k)}}\right\}$.

It can be solved by adapting the algorithm in (Friedman, Hastie and Tibshirani, 2010).

\section{Supplementary 3. Additional figures for application to cancer} genomic data.

\section{References.}

Boyd, S., Parikh, N., Chu, E., Peleato, B. and Eckstein, J. (2011). Distributed optimization and statistical learning via the alternating direction method of multipliers. Foundations and Trends in Machine Learning 3 1-122.

Friedman, J., Hastie, T. and Tibshirani, R. (2010). A note on the group lasso and a sparse group lasso. Technical Report. Department of Statistics, Stanford University, Stanford.

Hoefling, H. (2010). A path algorithm for the fused lasso signal approximator. Journal of Computational and Graphical Statistics 19 984-1006.
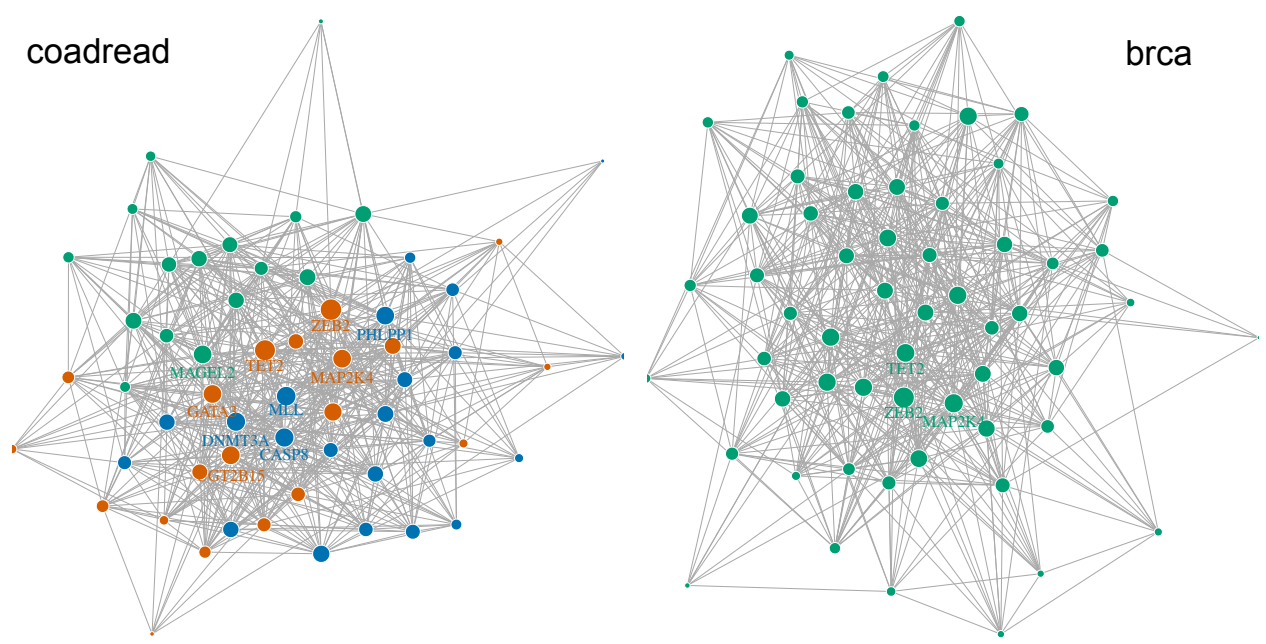

FIG 1. Tumor-specific modules for networks identified by DIG. The left panel: coadread; the right panel: brca. 

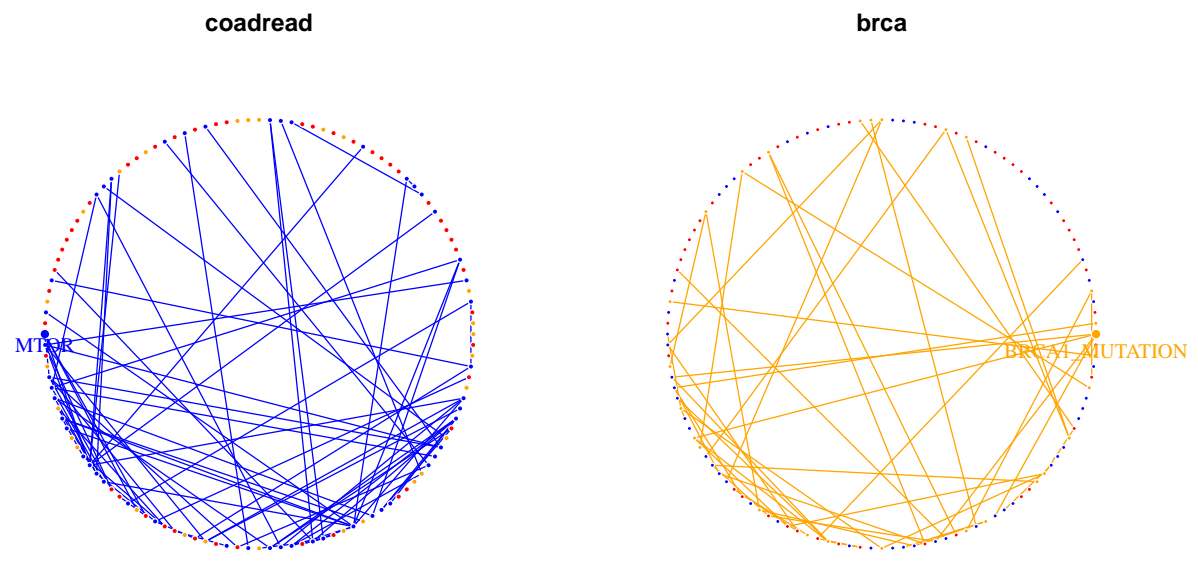

FIG 2. Tumor-specific networks identified by DIG. The left panel shows interactions presented in the coadread condition only, the right panel shows interactions presented in the brca condition only. Red nodes have the same degrees in the two conditions, blue nodes have higher degrees in the coadread condition, orange nodes have higher degrees in the brca condition. Some nodes are zoomed in as examples with their names shown in the pictures.

Department of Statistics

Institute for Systems Genomics

Center for Quantitative Medicine

Institute for Collaboration on Health, Intervention, and Policy

The Connecticut Institute for the Brain and Cognitive Sciences

UNIVERSITY OF CONNECTICUT

Storrs, Connecticut 06269

E-MAIL: yuping.zhang@uconn.edu

Department of Biostatistics

Yale School of Public Health

New Haven, Connecticut 06510

E-MAIL: hongyu.zhao@yale.edu
The Jackson Laboratory for Genomic Medicine Department of Biomedical EngineERING Department of Genetics and Genome Sciences Institute FOR Systems Genomics

University of Connecticut Farmington, Connecticut 06030 E-MAIL: zhengqing.ouyang@jax.org 